\title{
Effects of oregano essential oil on in vitro ruminal fermentation, methane production, and ruminal microbial community
}

\author{
Rui Zhou, ${ }^{1}$ Jianping Wu, ${ }^{1 *}$ Xia Lang, ${ }^{2}$ Lishan Liu, ${ }^{2}$ David P. Casper, ${ }^{3}$ Cailian Wang, ${ }^{2}$ Liping Zhang, ${ }^{1}$ \\ and Sheng $\mathrm{Wei}^{1}$ \\ ${ }^{1}$ College of Animal Science and Technology, Gansu Agricultural University, No. 1 Yingmen Village Anning, Lanzhou, Gansu, \\ People's Republic of China, 730070 \\ ${ }^{2}$ Animal Husbandry, Pasture, and Green Agriculture Institute, Gansu Academy of Agricultural Sciences, No. 1 Nongkeyuan Village Anning, \\ Lanzhou, Gansu, People's Republic of China, 730030 \\ ${ }^{3}$ Casper's Calf Ranch, 4890 West Lily Creek Road, Freeport, IL 61032
}

\section{ABSTRACT}

Different inclusion rates of oregano essential oil (OEO) were investigated for their effects on ruminal in vitro fermentation parameters, total gas, methane production, and bacterial communities. Treatments were (1) control, $0 \mathrm{mg} / \mathrm{L}$ of $\mathrm{OEO}(\mathrm{CON}) ; 13 \mathrm{mg} / \mathrm{L}$ (OEO1); $52 \mathrm{mg} / \mathrm{L}$ (OEO2); $91 \mathrm{mg} / \mathrm{L}$ (OEO3); and 130 $\mathrm{mg} / \mathrm{L}$ (OEO4), each incubated with $150 \mathrm{~mL}$ of buffered rumen fluid and 1,200 $\mathrm{mg}$ of substrate for $24 \mathrm{~h}$ using the Ankom in vitro gas production system (Ankom Technology Corp., Fairport, NY). Treatment responses were statistically analyzed using polynomial contrasts. Digestibility of DM, NDF, and ADF increased quadratically with increasing OEO inclusion rates. Digestibility of DM and NDF were highest for OEO2, whereas ADF digestibility was highest for OEO3, compared with CON, with the remaining treatments being intermediate and similar. Ammonia nitrogen concentrations decreased from $\mathrm{CON}$ at a quadratic rate with increasing $\mathrm{OEO}$ inclusion rates, and $\mathrm{OEO} 2$ had the lowest concentration compared with the other groups. Total VFA, acetate, propionate, butyrate, valerate, and isovalerate concentrations linearly decreased with increasing $\mathrm{OEO}$ inclusion rates. Total gas production levels by $\mathrm{CON}$ and $\mathrm{OEO} 4$ were greater than those of OEO1, OEO2, and OEO3 in a quadratic response, and methane production linearly decreased from CON, compared with $\mathrm{OEO} 4$, at a decreasing rate with $\mathrm{OEO}$ inclusion rates. As determined by $16 \mathrm{~S}$ rRNA sequencing, the $\alpha$ biodiversity of ruminal bacteria was similar among OEO inclusion rates. Increasing OEO inclusion rates linearly increased the relative abundance of Prevotella and Dialister bacteria. Several bacteria demonstrated dif-

Received March 13, 2019.

Accepted November 7, 2019.

*Corresponding author: wujp@gsagr.ac.cn ferent polynomial responses, whereas several bacteria were similar among increasing OEO inclusion rates. These results suggested that OEO supplementation can modify ruminal fermentation to alter VFA concentrations and reduce methane emissions by extensively altering the ruminal bacterial community, suggesting an optimal feeding rate for future animal studies of approximately $52 \mathrm{mg} / \mathrm{L}$ for mature ruminants.

Key words: bacterial composition, methane production, oregano essential oil, rumen fermentation, 16S rRNA

\section{INTRODUCTION}

Ruminant livestock production has been identified as a significant contributor to greenhouse gas emissions (Gerber et al., 2013). Seventy-one percent of the methane produced by enteric fermentation originates from ruminal fermentation (Gerber et al., 2013). In recent years, numerous strategies to mitigate methane emissions through the modulation of ruminal fermentation have been reported. Feeding natural feed additives has shown promising results (Hristov et al., 2013; Knapp et al., 2014). Among these natural additives, most are sourced from several plant types as secondary metabolites that have been reported to be useful in animal feeding (Wallace, 2004; Kamra et al., 2006), such as saponins, tannins, and essential oils (EO), which have demonstrated antimicrobial activities (Bakkali et al., 2008). Among these secondary metabolites, EO get the most attention and are being investigated as alternatives to antibiotics in animal nutrition (Greathead, 2003; Froehlich et al., 2017). In addition, EO and their bioactive compounds have been confirmed to modify ruminal fermentation by enhancing the efficiency of energy utilization while decreasing methane emissions (Tekippe et al., 2010; Joch et al., 2016). Essential oils have also been investigated as potential modifiers of ruminal biohydrogenation of dietary lipids, with the goal 
of enhancing healthful characteristics of milk and meat (Durmic et al., 2008; Lourenço et al., 2008).

Oregano (Origanum vulgare L.) is an herb with higher antioxidant capacity than other medicinal herbs (Dragland et al., 2003; Matsuura et al., 2003). The primary constituents of oregano EO (OEO) are carvacrol, $\gamma$-terpinene, thymol, p-cimene, and linalool, depending on origin and type of OEO (Sivropoulou et al.,1996; Baser, 2002). Oregano EO has been reported to have the second-highest oxygen radical absorbance capacity compared with clove (the highest), followed by cinnamon, ginger, and rosemary EO (Bentayeb et al., 2009). Thymol is a monoterpene with strong antimicrobial activity against a wide range of gram-positive and gram-negative bacteria and is one of the most actively researched EO (Burt, 2004). Carvacrol is a phenolic compound similar to thymol found in oregano, which also has strong antimicrobial activity. Busquet et al. (2005b) reported that carvacrol $(2.2 \mathrm{mg} / \mathrm{L})$ decreased large-peptide concentrations and increased ammonia nitrogen concentrations $2 \mathrm{~h}$ after feeding, using an in vitro long-term ruminal continuous culture system. Wang et al. (2009) evaluated a commercial OEO preparation (active chemical compounds: carvacrol, thymol) that demonstrated approximately a $12 \%$ decrease in methane production in sheep, similar in magnitude to methane production levels with flavomycin and Yucca schidigera (i.e., saponins). Oregano leaf material as an anti-methanogenic plant product, with no adverse effects on ruminal fermentation or NDF degradability, has been reported in vitro (Tekippe et al., 2010). Oregano EO was tested using a single dose and reduced ruminal methane production by positively improving total-tract apparent digestibility of dietary nutrients (Liu et al., 2017).

The study objectives were to verify our previous results and to evaluate inclusion rates of OEO to aid in determining the optimal feeding rate. Therefore, the specific study objective was to evaluate the effects of increasing OEO supplementation rates on ruminal in vitro fermentation, methane production, and ruminal bacterial communities. The hypothesis was that OEO would reduce fermentor methane production while improving feed digestibility and fermentation characteristics.

\section{MATERIALS AND METHODS}

\section{Feeding and Management}

All animal-handing protocols were approved by the Gansu Agricultural University Animal Care and Use Committee Guidelines (approved ID: 2012-2-159), fol-
Table 1. Ingredient and nutrient composition of the experimental ration (DM basis)

\begin{tabular}{lrlc}
\hline Ingredient & $\%$ & Nutrient level & $\%$ \\
\hline Corn silage & 65.50 & $\mathrm{DE}^{2}(\mathrm{MJ} / \mathrm{kg})$ & 14.2 \\
Corn & 25.74 & $\mathrm{CP}$ & 15.1 \\
Soybean meal & 2.34 & $\mathrm{NDF}$ & 51.2 \\
Rapeseed meal & 2.34 & $\mathrm{ADF}$ & 38.0 \\
Cottonseed meal & 2.34 & $\mathrm{Ca}$ & 0.41 \\
Limestone & 0.58 & $\mathrm{P}$ & 0.27 \\
NaCl & 0.58 & & \\
1\% Premix & 0.58 & & \\
Total & 100 & & \\
\end{tabular}

${ }^{1}$ The premix provided the following per $\mathrm{kg}$ of diet: vitamin A 220,000 $\mathrm{IU}$, vitamin $\mathrm{D}_{3} 72,000 \mathrm{IU}$, vitamin E 2,000 IU, D-biotin $40.0 \mathrm{mg}$, nicotinic acid amide 2,000 $\mathrm{mg}, \mathrm{Mn}$ (as manganese sulfate) $710 \mathrm{mg}$, Zn (as zinc sulfate) 2,005 mg, Fe (as ferrous sulfate) $830.0 \mathrm{mg}, \mathrm{Cu}$ (as copper sulfate) $680.0 \mathrm{mg}$, Co (as cobalt sulfate) $12 \mathrm{mg}$.

${ }^{2} \mathrm{DE}=$ digestive energy.

lowing the Chinese Standards for the Use and Care of Research Animals (He et al., 2016). Three German Merino sheep $\times$ local sheep crossbreed rams (initial live $\mathrm{BW}=56.68 \pm 2.14 \mathrm{~kg}$ ) fitted with permanent rumen cannulas were used as ruminal inoculum donors. Sheep were selected as the inoculum source due to being readily available, and Yáñez-Ruiz et al. (2016) indicated no differences between cattle and sheep when conducting in vitro experiments. Cows were offered a 65.5:34.5 forage-to-concentrate ratio (DM basis, \%) based on corn silage, ground corn, protein sources (soybean, rapeseed, and cottonseed meals), minerals, and vitamins (Table 1). The nutrient composition of the diet (\%,DM basis) was approximately $15.1 \% \mathrm{CP}$ and $51.2 \% \mathrm{NDF}$ (Table 1 ), to meet or exceed the nutrient requirements guidelines for sheep (NRC, 2007). Sheep were fed twice daily at 0800 and $1800 \mathrm{~h}$, before and throughout the duration of the experiment, and were given ad libitum access to water.

\section{Oregano Essential Oil Treatments}

The OEO was supplied as a dry granular powder (Rum-A-Fresh, Ralco Inc., Marshall, MN) containing approximately 1.3\% OEO, along with lactic acid, cobalt carbonate, and clinoptilolite as a carrier. Five inclusion rates of OEO were evaluated: $0(\mathbf{C O N}), 13$ $\mathrm{mg} / \mathrm{L}$ (OEO1), $52 \mathrm{mg} / \mathrm{L}$ (OEO2), $91 \mathrm{mg} / \mathrm{L}$ (OEO3), and $130 \mathrm{mg} / \mathrm{L}(\mathbf{O E O} 4)$, adjusted for the amount of OEO in the dry granular product added to the in vitro fermentation medium.

\section{In Vitro Fermentations}

Whole ruminal contents were obtained approximately $2 \mathrm{~h}$ after the morning feeding from 3 sheep in 
equal proportions, transported to the laboratory in a prewarmed and sealed flask, and immediately mixed. The ruminal fluid for incubations was obtained after filtering through 4 layers of $100 \mathrm{~mm} \times 100 \mathrm{~mm}$ medical gauze (Winner Inc. Ltd., Shenzhen, China) under constant $\mathrm{CO}_{2}$ flushing.

The in vitro experiment was carried out using the Ankom RFS gas production system (Ankom Technology Corp., Fairport, NY). The system comprised 24 fermentors having a $250-\mathrm{mL}$ volume, containing 1,200 mg of feed substrate (same ration fed to donor sheep, ground to pass through a 1-mm sieve) and the corresponding OEO inclusion rate. Each treatment had 4 bottles and 4 blank bottles (rumen fluid only) with no feed substrate, to correct for background gas production. Each fermentor was filled with $150 \mathrm{~mL}$ of ruminal inoculum, prepared as a 1:2 ratio of rumen fluid and artificial saliva. The artificial saliva was prepared anaerobically as described by Menke and Steingass (1988). All fermentors were incubated at $39^{\circ} \mathrm{C}$ for 24 $\mathrm{h}$ in a mild shaking water bath (SPH-110X24, Shiping Ltd., Shanghai, China).

\section{Sampling}

Headspace gas $(5 \mathrm{~mL})$ was collected from each fermentor bottle at $24 \mathrm{~h}$, using a sealed gas injection needle, for determination of methane concentrations. At the end of the 24-h incubation, fermentors were opened and $\mathrm{pH}$ was immediately measured using a $\mathrm{pH}$ meter and glass electrode (Type CG 842, Blueline 14 $\mathrm{pH}$, Schott Instruments, Weilheim, Germany). A liquid sub-sample was collected from each fermentor and stored in microcentrifuge tubes for later determination of VFA, ammonia nitrogen $\left(\mathrm{NH}_{3}-\mathrm{N}\right)$, microbial crude protein (MCP), and bacterial community determinations. Samples were stored at $-20^{\circ} \mathrm{C}$ for VFA and ammonia nitrogen and at $-80^{\circ} \mathrm{C}$ for $\mathrm{MCP}$ and bacterial community analyses, respectively, until analyzed. The residues of each fermentor were collected after centrifugation at $4,000 \times g$ for 10 min at room temperature to determine the DM, NDF, and ADF digestibility (TGL16, Cence Ltd., Changsha, China).

\section{Chemical Analysis}

The VFA concentrations were analyzed using a gas chromatograph (GC-2010, Shimadzu, Kyoto, Japan) following the procedures described by $\mathrm{Hu}$ et al. (2005). The $\mathrm{NH}_{3}-\mathrm{N}$ concentration was analyzed spectrophotometrically (UV-120-01, Shimadzu) using the procedures described by Chaney and Marbach (1962). The MCP was determined following the procedures of Lin et al. (2013a). Feed substrate and residues DM were determined following AOAC International (2016) procedures. The NDF and ADF in the feed substrate and residues were determined according to the methods described by Van Soest et al. (1991). Methane production was analyzed via gas chromatograph (GC-2010, Shimadzu) equipped with a flame ionization detector (FID-2010, Shimadzu; Hu et al., 2005). The column (HP-Innowax, 19091N-133, Agilent Technologies, Santa Clara, CA) of the GC was $30 \mathrm{~m} \times 0.25 \mathrm{~mm} \times 0.25 \mu \mathrm{m}$ in size, and the temperature was $80^{\circ} \mathrm{C}$. The gas flow rates for nitrogen, hydrogen, and air were 30,25 , and $400 \mathrm{~mL} / \mathrm{min}$, respectively. The front inject port, column oven, and detector temperatures were 50,375 , and $200^{\circ} \mathrm{C}$, respectively. The gas pressure was automatically recorded during incubation (Ankom Technology Corp.). The total gas production at $24 \mathrm{~h}$ was calculated based on the ideal gas equation (Ankom operations manual):

$$
\mathrm{V}_{\mathrm{x}}=\mathrm{P}_{\mathrm{kPa}} \times 1.3815886,
$$

where $\mathrm{V}_{\mathrm{x}}(\mathrm{mL})=$ gas volume at $39^{\circ} \mathrm{C}$, and $\mathrm{P}_{\mathrm{kPa}}=$ gas pressure.

\section{DNA Extraction, PCR Amplification, and 16S rRNA Sequencing}

Total genomic DNA from ruminal samples stored at $-80^{\circ} \mathrm{C}$ were extracted using the EZNA Stool DNA Kit (D4015, Omega Bio-tek Inc., Norcross, GA), following the manufacturer's instructions. The V3 to V4 region of the prokaryotic (bacterial and archaeal) small-subunit (16S) rRNA genes were amplified using slightly modified universal primer versions $338 \mathrm{~F}$ (5'-ACTCCTACGGGAGGCAGCAG-3') and 806R (5'-GGACTACHVGGGTWTCTAAT-3'; Fadrosh et al., 2014), where the barcode is an 8-base sequence unique to each sample on the $5^{\prime}$ primer ends and sequencing universal primers. Amplification via PCR was performed in a total volume of $25 \mu \mathrm{L}$ of reaction mixture containing 25 ng of template DNA, 12.5 $\mu \mathrm{L}$ of PCR Premix, $2.5 \mu \mathrm{L}$ of each primer, and PCR-grade water to adjust the volume. The PCR conditions to amplify the prokaryotic $16 \mathrm{~S}$ fragments consisted of an initial denaturation at $98^{\circ} \mathrm{C}$ for $30 \mathrm{~s} ; 35$ cycles of denaturation at $98^{\circ} \mathrm{C}$ for 10 $\mathrm{s}$, annealing at $54^{\circ} \mathrm{C}$ for $30 \mathrm{~s}$, and extension at $72^{\circ} \mathrm{C}$ for $45 \mathrm{~s}$; and then final extension at $72^{\circ} \mathrm{C}$ for $10 \mathrm{~min}$. The PCR products were confirmed using $2 \%$ agarose gel electrophoresis. The PCR products were purified using AMPure XT beads (Beckman Coulter Genomics, Danvers, MA) and quantified by Qubit (Invitrogen, Carls- 
bad, CA). Amplicon sequencing was conducted on an Illumina MiSeq $2 \times 3$ platform to generate overlapping paired-end $2 \times 300$ bp (Bionew Ltd., Hohhot, China).

\section{Bioinformatics and Analyses}

All reads were processed and analyzed using the following procedures. First, paired-end reads were assigned to samples based on their unique barcode and truncated by cutting off the barcode and primer sequence. Paired-end reads were then merged using FLASH 1.2.7 (Magoč and Salzberg, 2011). Quality filtering of the raw tags was performed under specific filtering conditions to obtain high-quality clean tags according to the fqtrim (version 0.94, National Center for Biotechnology Information, Bethesda, MD). Chimeric sequences were filtered using USEARCH software based on the UCHIME algorithm (Edgar et al., 2011). Bacterial operational taxonomic units (OTU) were selected using USEARCH (version 2.3.4; Edgar, 2010), applying $97 \%$ sequence similarity thresholds. Representative sequences for each OTU were chosen using Quantitative Insights Into Microbial Ecology (QIIME; Wemheuer et al., 2014) programs (version 1.9.1), which were assigned from the Ribosomal Database Project for taxonomic classification using the BLAST approach (Mitra et al., 2011). Alpha diversity analysis, including abundancebased coverage estimators (ACE), Good's coverage, Chao1, Shannon, Simpson, and observed OTU indices, were calculated with QIIME 1.9.0 according to Wemheuer et al. (2014).

\section{Statistical Analyses}

All data were checked for normality and outliers using the UNIVARIATE procedure of SAS (version 9.4, SAS Institute Inc., Cary, NC) before any statistical analyses were conducted. We performed ANOVA using the $\mathrm{R}$ package (version 3.4.1, R Core Team, 2017) or SAS. The Mann-Whitney U test was applied to determine the significance of $\alpha$ diversity (Zeng et al., 2015). The data of ruminal fermentation parameters and nutrient digestibility were statistically analyzed using the PROC MIXED procedure of SAS. The statistical model used was as follows: $Y_{i j}=\mu+R_{i}+T_{j}+e_{i j}$, where $Y_{i j}=$ dependent variable, $\mu=$ overall mean, $R_{i}=$ replication, $T_{j}$ $=$ treatment, and $\mathrm{e}_{\mathrm{ij}}=$ random error. Treatment effects were considered fixed, and replication was considered to be random effect. Polynomial contrasts were used to test the linear, quadratic, cubic, and quartic effects of treatments (i.e., OEO inclusion rate). Differences among treatments were separated using the PDIFF statement of SAS. Statistical significance was set to $P$
$<0.05$, and a tendency of difference was declared at $P$ $<0.10$.

\section{RESULTS}

\section{Effects on Rumen Fermentation Characteristics}

The digestibility of DM, NDF, and ADF increased quadratically $(P<0.03)$ with increasing OEO inclusion rates (Table 2). In slight contrast, DM and NDF digestibility was highest for $\mathrm{OEO} 2$, whereas ADF digestibility was highest for OEO3 compared with CON, with the remaining treatments being intermediate and similar $(P>0.05)$. It thus appears that OEO3 would be the optimal feeding rate for future in vivo studies.

Fermentor $\mathrm{pH}$ demonstrated a linear $(P<0.05)$ increase with increasing $\mathrm{OEO}$ inclusion rate, with the highest $\mathrm{pH}$ observed for the highest inclusion rate of OEO (OEO4). In contrast with fermentor $\mathrm{pH}, \mathrm{NH}_{3}-\mathrm{N}$ concentrations demonstrated a quartic response $(P<$ 0.01) with increasing OEO inclusion rates, such that OEO2 had the lowest $(P<0.05) \mathrm{NH}_{3}-\mathrm{N}$ concentrations compared with the CON and OEO1 fermentors. Inclusion rate of OEO demonstrated mixed effects on fermentor VFA concentrations (Table 2). Microbial $\mathrm{CP}$ concentrations demonstrated a quadratic response $(P<0.10)$, such that the OEO2 fermentor had the highest MCP concentration compared with fermentors CON, OEO3, and OEO4. The MCP concentrations were inverse of the fermentor ammonia concentrations, indicating that ammonia was being incorporated into the synthesis of MCP.

Total concentrations of VFA, propionate, butyrate, isovalerate, and acetate-to-propionate ratio demonstrated trends $(P<0.10)$ or significant $(P<0.05)$ quartic responses to increasing OEO inclusion rate, decreasing to lower concentrations for OEO4 compared with CON fermentors. Acetate demonstrated a quadratic response $(P<0.10)$, with $\mathrm{OEO} 4$ decreasing to a lower concentration compared with fermentors $\mathrm{CON}$ or OEO1. Isobutyrate concentrations linearly decreased $(P<0.01)$ with increasing $\mathrm{OEO}$ inclusion rate. As the first OEO inclusion rate was added to the fermentors, most VFA remained similar $(P>0.10)$ to CON, but further increases in OEO inclusion rate decreased VFA concentrations $(P<0.05)$, meaning that very high OEO inclusion rates can become detrimental or inhibitory to VFA production.

\section{Effects on Total Gas and Methane Production}

Total 24-h gas production demonstrated a quartic $(P<0.10)$ response, decreasing to lowest for OEO2 
Table 2. Fermentor nutrient digestibility, $\mathrm{pH}$, ammonia $\mathrm{N}\left(\mathrm{NH}_{3}-\mathrm{N}\right)$, microbial crude protein (MCP), VFA, and gas production for fermentors fed a control $(\mathrm{CON})$ ration supplemented with increasing levels of oregano essential oil (OEO)

\begin{tabular}{|c|c|c|c|c|c|c|c|c|c|c|}
\hline \multirow[b]{2}{*}{ Measurement } & \multicolumn{5}{|c|}{ OEO $\operatorname{dose}^{1}(\mathrm{mg} / \mathrm{L})$} & \multirow[b]{2}{*}{ SEM } & \multicolumn{4}{|c|}{ Contrast $^{2}(P<)$} \\
\hline & $\mathrm{CON}$ & OEO1 & $\mathrm{OEO} 2$ & OEO3 & OEO4 & & $\mathrm{L}$ & $\mathrm{Q}$ & $\mathrm{C}$ & $\mathrm{Qu}$ \\
\hline $\mathrm{DMD}^{3}$ & $38.9^{\mathrm{b}}$ & $41.2^{\mathrm{ab}}$ & $44.9^{\mathrm{a}}$ & $44.8^{\mathrm{a}}$ & $41.4^{\mathrm{ab}}$ & 1.60 & 0.13 & $<0.01$ & 0.90 & 0.97 \\
\hline $\mathrm{NDFD}^{4}$ & $26.3^{\mathrm{b}}$ & $28.2^{\mathrm{ab}}$ & $32.0^{\mathrm{a}}$ & $29.5^{\mathrm{ab}}$ & $27.8^{\mathrm{b}}$ & 1.35 & 0.42 & 0.01 & 0.25 & 0.54 \\
\hline $\mathrm{ADFD}^{5}$ & $25.6^{\mathrm{b}}$ & $27.0^{\mathrm{ab}}$ & $28.1^{\mathrm{ab}}$ & $29.5^{\mathrm{a}}$ & $25.4^{\mathrm{b}}$ & 1.53 & 0.77 & 0.03 & 0.47 & 0.50 \\
\hline $\mathrm{pH}$ & $5.54^{\mathrm{b}}$ & $5.59^{\mathrm{b}}$ & $5.60^{\mathrm{b}}$ & $5.67^{\mathrm{b}}$ & $5.72^{\mathrm{a}}$ & 0.05 & $<0.01$ & 0.89 & 0.47 & 0.40 \\
\hline \multicolumn{11}{|l|}{$\mathrm{VFA}(\mathrm{mmol} / \mathrm{L})$} \\
\hline Total & $162.5^{\mathrm{a}}$ & $161.4^{\mathrm{a}}$ & $155.3^{\mathrm{b}}$ & $147.8^{\mathrm{c}}$ & $145.6^{\mathrm{d}}$ & 1.71 & $<0.01$ & 0.29 & 0.13 & 0.07 \\
\hline Acetate & $106.9^{\mathrm{a}}$ & $104.8^{\mathrm{a}}$ & $98.5^{\mathrm{b}}$ & $97.0^{\mathrm{b}}$ & $95.5^{\mathrm{b}}$ & 0.86 & $<0.01$ & $<0.01$ & 0.26 & 0.32 \\
\hline Propionate & $44.9^{\mathrm{b}}$ & $46.2^{\mathrm{ab}}$ & $48.3^{\mathrm{a}}$ & $43.6^{\mathrm{b}}$ & $43.3^{\mathrm{b}}$ & 1.13 & 0.03 & 0.02 & $<0.01$ & 0.06 \\
\hline Butyrate & $6.41^{\mathrm{a}}$ & $5.81^{\mathrm{b}}$ & $5.30^{\mathrm{bc}}$ & $4.12^{\mathrm{c}}$ & $4.01^{\mathrm{cd}}$ & 0.70 & $<0.01$ & 0.09 & 0.49 & 0.03 \\
\hline Valerate & $1.34^{\mathrm{ab}}$ & $1.45^{\mathrm{a}}$ & $1.01^{\mathrm{b}}$ & $0.94^{\mathrm{b}}$ & $0.80^{\mathrm{b}}$ & 0.08 & $<0.01$ & 0.21 & 0.65 & 0.36 \\
\hline Isobutyrate & $0.77^{\mathrm{a}}$ & $0.72^{\mathrm{a}}$ & $0.50^{\mathrm{ab}}$ & $0.46^{\mathrm{ab}}$ & $0.37^{\mathrm{b}}$ & 0.08 & $<0.01$ & 0.46 & 0.66 & 0.48 \\
\hline
\end{tabular}

\footnotetext{
${ }^{\mathrm{a}-\mathrm{d}}$ Means within a row with unlike superscripts differ $(P<0.05)$.

${ }^{1}$ Data were analyzed using OEO dose levels of 0 (CON), $13 \mathrm{mg} / \mathrm{L}$ (OEO1), $52 \mathrm{mg} / \mathrm{L}$ (OEO2), $91 \mathrm{mg} / \mathrm{L}$ (OEO3), or 130 mg/L (OEO4).

${ }^{2}$ Probability of contrast being less than the value in the column; $\mathrm{L}=$ linear; $\mathrm{Q}=$ quadratic; $\mathrm{C}=$ cubic; $\mathrm{Qu}=$ quartic.

${ }^{3} \mathrm{DMD}=$ dry matter digestibility.

${ }^{4} \mathrm{NDFD}=$ neutral detergent fiber digestibility.

${ }^{5} \mathrm{ADFD}=$ acid detergent fiber digestibility.
}

then increasing with increasing OEO inclusion rate (Table 2), whereas 24-h total methane production and methane/total gas were linearly decreased $(P<0.05)$ with increasing $\mathrm{OEO}$ inclusion rate, such that OEO3 had the lowest methane production and methane/total gas compared with the CON and OEO1 fermentors. However, as total gas and methane decreased, methane as a percentage of total gas production declined faster as the OEO inclusion rate increased. Methane decreasing at a greater rate than total gas production should direct more energy into VFA production.

\section{Effects on Rumen Bacterial Community}

A total of 379,768 raw bacterial $16 \mathrm{~S}$ rRNA gene sequences were obtained. After quality control and filtering, 25,317 valid sequences were analyzed for each sample. A total of 4,410 OTU based on $97 \%$ sequence similarity were generated. The $\alpha$ diversity of $16 \mathrm{~S}$ rRNA gene OTU was not altered by increasing OEO inclusion rate $(P>0.10$; Table 3$)$, except for Chao1, which demonstrated a linear $(P<0.05)$ reduction such that OEO3 fermentors were lowest $(P<0.05)$ compared with remaining fermentors.

Based on the obtained OTU, a total of 24 genera were observed to have a relative abundance greater than 1\% (Figure 1). The most abundant microbial genus detected via $16 \mathrm{~S}$ rRNA gene sequence data was Prevotella, which demonstrated a linear $(P<0.05)$ response, with increasing $\mathrm{OEO}$ inclusion rate being greatest $(P<0.05)$ for OEO4 fermentors compared with CON fermentors and the remaining treatments being intermediate (Table 4). Several microbial genera were similar $(P>0.05)$ among all OEO inclusion rates. Firmicutes unclassified, Eubacterium, Lachnospiraceae unclassified, Bifidobacterium, and Oxobacter demonstrated quartic $(P<0.05)$ responses with increasing OEO inclusion rates that were not consistent across microbial genera. Firmicutes unclassified, Eubacterium, Bifidobacterium, and Oxobacter were lower for OEO1 fermentors compared with CON fermentors, whereas Lachnospiraceae unclassified was lower for $\mathrm{CON}, \mathrm{OEO} 2$, and OEO3 fermentors compared with OEO1 fermentors, with the remaining treatments being intermediate. The microbial genera with more than $2 \%$ relative abundance demonstrate greater relative abundance of Prevotella and Dialister, while reducing $(P<0.010)$ Veillonellaceae unclassified, Porphyromonadaceae unclassified, and Firmicutes unclassified via linear, quadratic, cubic, or quartic responses with increasing OEO inclusion rate compared with CON. 


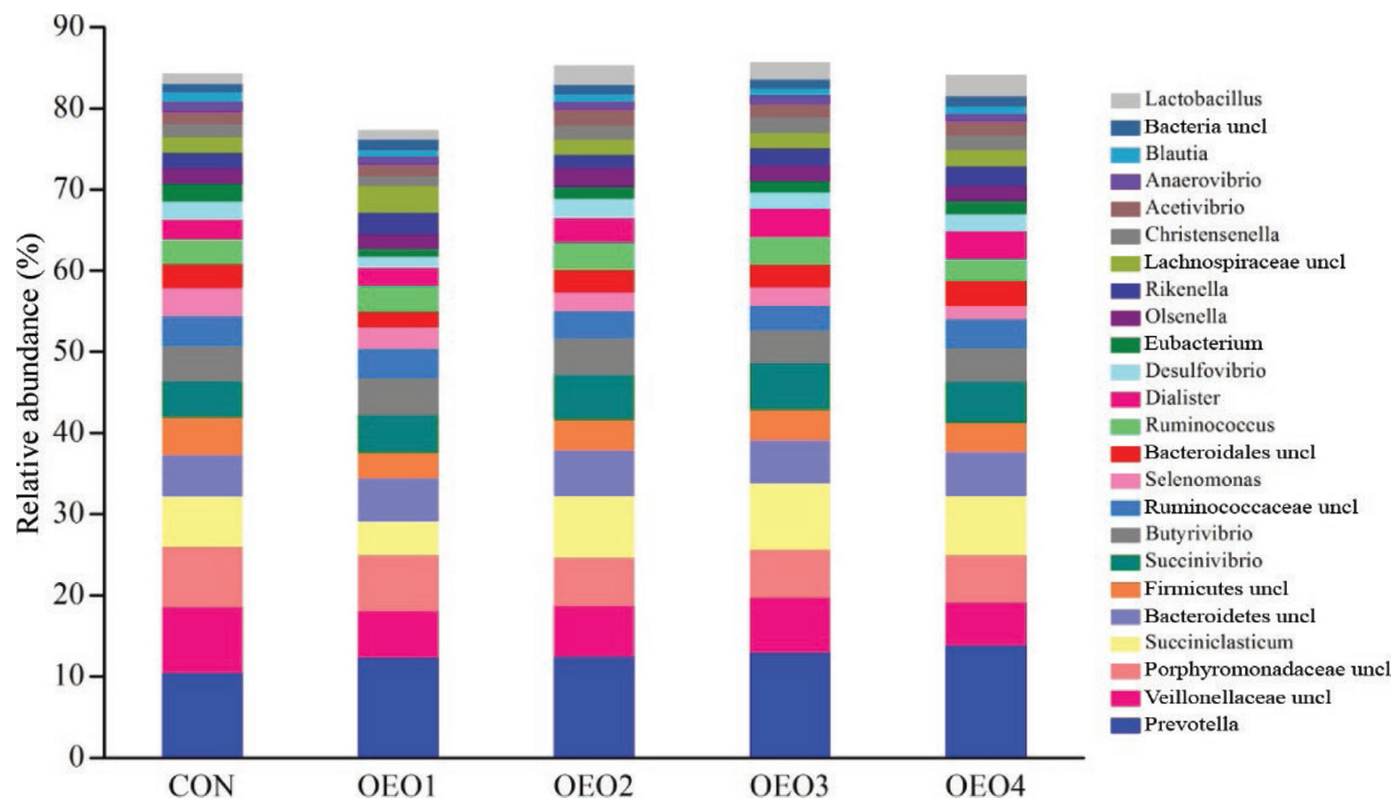

Figure 1. Ruminal bacterial community composition of genera with a relative abundance greater than $1 \%$. Data were analyzed using inclusion rates of oregano essential oil $(\mathrm{OEO})$ of $0(\mathrm{CON}), 13 \mathrm{mg} / \mathrm{L}(\mathrm{OEO}), 52 \mathrm{mg} / \mathrm{L}(\mathrm{OEO} 2), 91 \mathrm{mg} / \mathrm{L}(\mathrm{OEO})$, and $130 \mathrm{mg} / \mathrm{L}(\mathrm{OEO} 4)$. Uncl = unclassified.

\section{DISCUSSION}

\section{Effects on Rumen Fermentation Characteristics}

For a ruminant animal, most of the DM and fiber of a feed or ration is ruminally digested. Therefore, ruminal $\mathrm{DM}$ and fiber digestibility are appropriate indices for evaluating OEO effects (Lin et al., 2009). In the literature, the $\mathrm{EO}$ effects on nutrient digestibility by the rumen microbiome have been mixed. Some researchers have suggested that EO have no effects on fiber degradation but can reduce the degradation of readily degradable substrates, such as protein and starch, ow- ing to OEO inhibition of amylolytic and proteolytic bacteria (Wallace et al., 2002; Hart et al., 2008). On the contrary, other authors have reported positive effects (Benchaar et al., 2006; Yang et al., 2007) or no effects (Meyer et al., 2009; Sallam et al., 2009) of EO on ruminal digestibility. In the current study, supplementation of $52 \mathrm{mg} / \mathrm{L}$ and $91 \mathrm{mg} / \mathrm{L}$ OEO increased in vitro fermentor DM, ADF, and NDF digestibility (Table 2) compared with CON, with digestibility increasing with increasing $\mathrm{OEO}$ inclusion rate to a point of being maximal at 52 or $91 \mathrm{mg} / \mathrm{L}$. These results corresponded to a linear decrease in VFA production, to the extent that a high $\mathrm{OEO}$ inclusion rate can be detrimental by

Table 3. Changes in richness and diversity of the ruminal bacterial community in fermentors fed a control (CON) or ration supplemented with increasing inclusion rates of oregano essential oil (OEO)

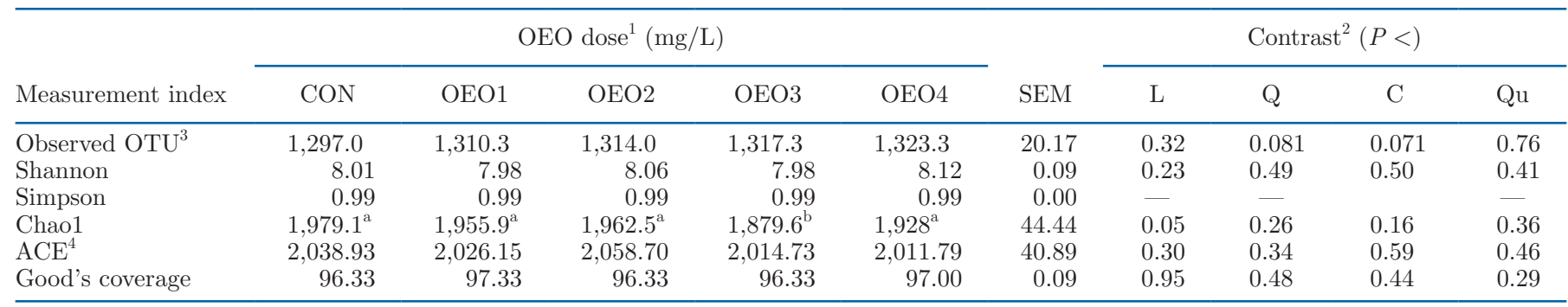

${ }_{\mathrm{a}, \mathrm{b}}$ Means within a row with unlike superscripts differ $(P<0.05)$.

${ }^{1}$ Data were analyzed using OEO dose levels of 0 (CON), $13 \mathrm{mg} / \mathrm{L}$ (OEO1), $52 \mathrm{mg} / \mathrm{L}$ (OEO2), $91 \mathrm{mg} / \mathrm{L}$ (OEO3), or 130 mg/L (OEO4).

${ }^{2}$ Probability of a contrast being less than value in column; $\mathrm{L}=$ linear; $\mathrm{Q}=$ quadratic; $\mathrm{C}=$ cubic; $\mathrm{Qu}=$ quartic. Dashes indicate no statistical test because all values $=0.99$.

${ }^{3} \mathrm{OTU}=$ operational taxonomic unit.

${ }^{4} \mathrm{ACE}=$ abundance-based coverage estimator. 
Table 4. Relative abundances of microbial genera (\% of total sequences) of the microbial community in fermentors fed a control (CON) ration with increasing inclusion rates of oregano essential oil (OEO)

\begin{tabular}{|c|c|c|c|c|c|c|c|c|c|c|}
\hline \multirow[b]{2}{*}{ Genus } & \multicolumn{5}{|c|}{ OEO dose ${ }^{1}(\mathrm{mg} / \mathrm{L})$} & \multirow[b]{2}{*}{ SEM } & \multicolumn{4}{|c|}{ Contrast $^{2}(P<)$} \\
\hline & $\mathrm{CON}$ & OEO1 & $\mathrm{OEO} 2$ & OEO3 & OEO4 & & $\mathrm{L}$ & $\mathrm{Q}$ & $\mathrm{C}$ & $\mathrm{Qu}$ \\
\hline Prevotella & $10.54^{\mathrm{b}}$ & $12.43^{\mathrm{ab}}$ & $12.58^{\mathrm{ab}}$ & $13.03^{\mathrm{a}}$ & $13.88^{\mathrm{a}}$ & 1.03 & 0.05 & 0.64 & 0.35 & 0.42 \\
\hline Veillonellaceae uncl $^{3}$ & $8.12^{\mathrm{a}}$ & $5.76^{\mathrm{b}}$ & $6.21^{\mathrm{ab}}$ & $6.82^{\mathrm{ab}}$ & $5.36^{\mathrm{b}}$ & 0.64 & 0.09 & 0.86 & 0.02 & 0.15 \\
\hline Porphyromonadaceae uncl & $7.45^{\mathrm{a}}$ & $6.87^{\mathrm{ab}}$ & $5.98^{\mathrm{ab}}$ & $5.92^{\mathrm{b}}$ & $5.83^{\mathrm{b}}$ & 0.60 & 0.02 & 0.25 & 0.59 & 0.96 \\
\hline Succiniclasticum & $6.17^{\mathrm{b}}$ & $4.14^{\mathrm{c}}$ & $7.56^{\mathrm{ab}}$ & $8.14^{\mathrm{a}}$ & $7.24^{\mathrm{ab}}$ & 0.61 & $<0.01$ & 0.05 & 0.04 & $<0.01$ \\
\hline Bacteroidetes uncl & 5.15 & 5.37 & 5.68 & 5.34 & 5.47 & 0.69 & 0.74 & 0.70 & 0.63 & 0.88 \\
\hline Firmicutes uncl & $4.58^{\mathrm{a}}$ & $3.10^{\mathrm{c}}$ & $3.71^{\mathrm{bc}}$ & $3.71^{\mathrm{ab}}$ & $3.59^{\mathrm{b}}$ & 0.44 & 0.29 & 0.30 & 0.10 & $<0.01$ \\
\hline Succinivibrio & 4.46 & 4.72 & 5.50 & 5.74 & 5.08 & 0.64 & 0.26 & 0.18 & 0.76 & 0.96 \\
\hline Butyrivibrio & 4.41 & 4.48 & 40.61 & 4.16 & 4.09 & 0.53 & 0.51 & 0.71 & 0.67 & 0.81 \\
\hline Ruminococcaceae uncl & 3.64 & 3.53 & 3.29 & 2.99 & 3.64 & 0.41 & 0.78 & 0.28 & 0.58 & 0.62 \\
\hline Selenomonas & $3.49^{\mathrm{a}}$ & $2.73^{\mathrm{a}}$ & $2.31^{\mathrm{bc}}$ & $2.23^{\mathrm{bc}}$ & $1.66^{\mathrm{c}}$ & 0.43 & $<0.01$ & 0.55 & 0.29 & 0.66 \\
\hline Bacteroidales uncl & 2.99 & 1.99 & 2.78 & 2.86 & 3.09 & 0.51 & 0.27 & 0.59 & 0.28 & 0.17 \\
\hline Ruminococcus & 2.95 & 3.14 & 3.28 & 3.30 & 2.59 & 0.57 & 0.49 & 0.22 & 0.79 & 0.85 \\
\hline Dialister & $2.43^{\mathrm{ab}}$ & $2.23^{\mathrm{b}}$ & $3.12^{\mathrm{a}}$ & $3.53^{\mathrm{a}}$ & $3.48^{\mathrm{a}}$ & 0.44 & 0.03 & 0.35 & 0.47 & 0.47 \\
\hline Desulfovibrio & 2.26 & 1.37 & 2.34 & 1.95 & 2.07 & 0.45 & 0.55 & 0.85 & 0.61 & 0.07 \\
\hline Eubacterium & $2.18^{\mathrm{a}}$ & $0.95^{\mathrm{b}}$ & $1.48^{\mathrm{a}}$ & $1.40^{\mathrm{ab}}$ & $1.63^{\mathrm{a}}$ & 0.32 & 0.75 & 0.13 & 0.12 & $<0.01$ \\
\hline Olsenella & 1.96 & 1.80 & 2.37 & 1.97 & 1.82 & 0.43 & 0.87 & 0.27 & 0.84 & 0.18 \\
\hline Rikenella & 1.91 & 2.64 & 1.65 & 2.17 & 2.49 & 0.42 & 0.57 & 0.23 & 0.69 & 0.09 \\
\hline Lachnospiraceae uncl & $1.91^{\mathrm{b}}$ & $3.27^{\mathrm{a}}$ & $1.87^{\mathrm{b}}$ & $1.84^{\mathrm{b}}$ & $2.03^{\mathrm{ab}}$ & 0.57 & 0.13 & 0.29 & 0.50 & 0.05 \\
\hline Christensenella & 1.61 & 1.21 & 1.71 & 1.91 & 1.78 & 0.41 & 0.33 & 0.74 & 0.45 & 0.34 \\
\hline Acetivibrio & 1.51 & 1.54 & 2.00 & 1.70 & 1.75 & 0.34 & 0.53 & 0.36 & 0.55 & 0.54 \\
\hline Anaerovibrio & 1.25 & 0.84 & 0.92 & 0.98 & 0.83 & 0.24 & 0.39 & 0.73 & 0.27 & 0.38 \\
\hline Blautia & 1.14 & 0.89 & 0.93 & 0.85 & 0.96 & 0.44 & 0.71 & 0.58 & 0.86 & 0.56 \\
\hline Bacteria uncl & 1.00 & 1.31 & 1.14 & 1.15 & 1.23 & 0.12 & 0.46 & 0.92 & 0.15 & 0.12 \\
\hline Lactobacillus & $1.06^{\mathrm{b}}$ & $0.94^{\mathrm{b}}$ & $2.17^{\mathrm{a}}$ & $1.93^{\mathrm{a}}$ & $2.44^{\mathrm{a}}$ & 0.33 & $<0.01$ & 0.43 & 0.48 & 0.12 \\
\hline Anaerovorax & 0.87 & 0.88 & 0.83 & 0.75 & 0.91 & 0.10 & 0.89 & 0.43 & 0.52 & 0.88 \\
\hline Clostridium & 0.83 & 0.74 & 0.82 & 0.80 & 0.80 & 0.11 & 0.96 & 0.99 & 0.77 & 0.48 \\
\hline Ethanoligenens & 0.69 & 0.56 & 0.76 & 0.65 & 0.73 & 0.14 & 0.58 & 0.94 & 0.92 & 0.31 \\
\hline Bulleidia & 0.64 & 0.54 & 0.62 & 0.49 & 0.50 & 0.11 & 0.17 & 0.97 & 0.84 & 0.28 \\
\hline Mitsuokella & $0.59^{\mathrm{a}}$ & $0.43^{\mathrm{b}}$ & $0.28^{\mathrm{bc}}$ & $0.23^{\mathrm{c}}$ & $0.15^{\mathrm{c}}$ & 0.07 & $<0.01$ & 0.06 & 0.20 & 0.69 \\
\hline Erysipelothrix & 0.58 & 0.40 & 0.44 & 0.45 & 0.44 & 0.10 & 0.55 & 0.45 & 0.33 & 0.33 \\
\hline Coriobacteriacease uncl & $0.51^{\mathrm{a}}$ & $0.34^{\mathrm{bc}}$ & $0.42^{\mathrm{a}}$ & $0.29^{\mathrm{c}}$ & $0.27^{\mathrm{c}}$ & 0.06 & 0.02 & 0.81 & 0.58 & 0.05 \\
\hline Saccharibacteria & $0.49^{\mathrm{bc}}$ & $0.35^{\mathrm{c}}$ & $0.60^{\mathrm{ab}}$ & $0.62^{\mathrm{a}}$ & $0.52^{\mathrm{bc}}$ & 0.08 & 0.08 & 0.07 & 0.12 & 0.13 \\
\hline Paraprevotella & $0.44^{\mathrm{b}}$ & $0.41^{\mathrm{b}}$ & $0.51^{\mathrm{b}}$ & $0.64^{\mathrm{ab}}$ & $0.80^{\mathrm{a}}$ & 0.11 & $<0.01$ & 0.41 & 0.77 & 0.74 \\
\hline Saccharofermentans & $0.39^{\mathrm{ab}}$ & $0.29^{\mathrm{b}}$ & $0.37^{\mathrm{ab}}$ & $0.40^{\mathrm{ab}}$ & $0.54^{\mathrm{a}}$ & 0.11 & 0.07 & 0.32 & 0.89 & 0.27 \\
\hline Schwartzia & 0.36 & 0.20 & 0.31 & 0.31 & 0.25 & 0.09 & 0.71 & 0.76 & 0.23 & 0.18 \\
\hline Oribacterium & 0.36 & 0.35 & 0.38 & 0.43 & 0.36 & 0.06 & 0.45 & 0.38 & 0.39 & 0.96 \\
\hline Alloprevotella & 0.34 & 0.27 & 0.36 & 0.33 & 0.41 & 0.07 & 0.27 & 0.71 & 0.96 & 0.28 \\
\hline Syntrophococcus & 0.31 & 0.24 & 0.27 & 0.25 & 0.29 & 0.05 & 0.91 & 0.27 & 0.56 & 0.13 \\
\hline Prevotellaceae uncl & 0.29 & 0.15 & 0.25 & 0.28 & 0.27 & 0.04 & 0.34 & 0.83 & 0.14 & 0.07 \\
\hline Moryella & 0.28 & 0.26 & 0.20 & 0.25 & 0.26 & 0.05 & 0.84 & 0.29 & 0.55 & 0.71 \\
\hline Erysipelotrichaceae uncl & $0.26^{\mathrm{a}}$ & $0.12^{\mathrm{b}}$ & $0.33^{\mathrm{a}}$ & $0.27^{\mathrm{a}}$ & $0.35^{\mathrm{a}}$ & 0.07 & 0.07 & 0.95 & 0.91 & $<0.01$ \\
\hline Lachnoclostridium & 0.25 & 0.45 & 0.18 & 0.22 & 0.23 & 0.07 & 0.25 & 0.41 & 0.37 & 0.10 \\
\hline Pyramidobacter & 0.20 & 0.15 & 0.15 & 0.17 & 0.12 & 0.05 & 0.38 & 0.98 & 0.21 & 0.77 \\
\hline Turicibacter & 0.19 & 0.18 & 0.20 & 0.17 & 0.18 & 0.06 & 0.82 & 0.93 & 0.75 & 0.65 \\
\hline Intestinimonas & 0.18 & 0.09 & 0.12 & 0.09 & 0.12 & 0.04 & 0.40 & 0.31 & 0.57 & 0.18 \\
\hline Desulfobulbus & 0.16 & 0.015 & 0.22 & 0.31 & 0.25 & 0.20 & 0.44 & 0.71 & 0.74 & 0.99 \\
\hline Oscillibacter & 0.15 & 0.61 & 0.17 & 0.21 & 0.18 & 0.48 & 0.48 & 0.96 & 0.43 & 0.37 \\
\hline Atopobium & 0.15 & 0.10 & 0.13 & 0.13 & 0.13 & 0.05 & 0.97 & 0.76 & 0.67 & 0.53 \\
\hline Roseburia & 0.14 & 0.46 & 0.19 & 0.21 & 0.22 & 0.37 & 0.74 & 0.99 & 0.52 & 0.46 \\
\hline Streptococcus & 0.14 & 0.09 & 0.31 & 0.23 & 0.26 & 0.25 & 0.47 & 0.72 & 0.91 & 0.48 \\
\hline Mogibacterium & 0.14 & 0.12 & 0.16 & 0.16 & 0.12 & 0.05 & 0.96 & 0.48 & 0.67 & 0.66 \\
\hline Oscillospira & 0.13 & 0.24 & 0.13 & 0.11 & 0.13 & 0.12 & 0.39 & 0.70 & 0.30 & 0.44 \\
\hline Fretibacterium & 0.13 & 0.08 & 0.21 & 0.18 & 0.24 & 0.07 & 0.09 & 0.74 & 0.78 & 0.09 \\
\hline Pseudomonas & 0.11 & 0.08 & 0.05 & 0.07 & 0.05 & 0.07 & 0.40 & 0.68 & 0.60 & 0.95 \\
\hline Bifidobacterium & $0.57^{\mathrm{bc}}$ & $0.46^{\mathrm{c}}$ & $0.97^{\mathrm{a}}$ & $0.81^{\mathrm{ab}}$ & $0.76^{\mathrm{ab}}$ & 0.14 & 0.10 & 0.05 & 0.87 & 0.04 \\
\hline Oxobacter & $0.34^{\mathrm{ab}}$ & $0.15^{\mathrm{b}}$ & $0.25^{\mathrm{a}}$ & $0.22^{\mathrm{ab}}$ & $0.26^{\mathrm{ab}}$ & 0.05 & 0.87 & 0.39 & 0.31 & 0.04 \\
\hline
\end{tabular}

${ }^{\mathrm{a}-\mathrm{c}}$ Means in the same row with unlike superscripts differ, $P<0.05$.

${ }^{1}$ Data were analyzed using OEO dose levels of 0 (CON), $13 \mathrm{mg} / \mathrm{L}$ (OEO1), $52 \mathrm{mg} / \mathrm{L}$ (OEO2), $91 \mathrm{mg} / \mathrm{L}$ (OEO3), or 130 mg/L (OEO4).

${ }^{2} \mathrm{~L}=$ linear; $\mathrm{Q}=$ quadratic; $\mathrm{C}=$ cubic; and $\mathrm{Qu}=$ quartic.

${ }^{3}$ Uncl $=$ unclassified. 
reducing VFA concentrations. Why digestibility could be increased with a decrease in VFA may be related to the production of microbial extracellular enzymes enhancing DM and fiber digestibility (Priest, 1977), but the hydrolysis products were not fermented to VFA. These findings are in contrast with the results reported by Tager and Krause (2011), who reported that high levels of EO negatively affected ruminal fiber digestibility, but do agree that they theoretically could reduce ruminal VFA production.

The potential uses of EO as a ruminal fermentation modifier have been reported in the literature (Molero et al., 2004; Simitzis, 2017). Numerous in vitro experiments have reported that different types and doses of EO could inhibit $\mathrm{NH}_{3}$ concentrations (Cardozo et al., 2005; Lin et al., 2012; Lin et al., 2013b). Hristov et al. (2013) also observed a decrease of $\mathrm{NH}_{3}$ and butyrate concentration when feeding dairy cows Origanum vulgare L. leaves $(250,500$, and $750 \mathrm{~g} / \mathrm{cow})$. The various EO available as natural feed additives could be considered useful in ruminant nutrition by reducing ruminal ammonia $\mathrm{N}$ concentrations and protein deamination by inhibiting hyper-ammonia producing (HAP) bacteria (Patra, 2011). The quartic response in decreasing ruminal ammonia nitrogen concentrations with increasing OEO inclusion rate observed in the present study likely resulted from the inhibition of proteolysis, peptidolysis, amino acid deamination, or an increase in microbial protein synthesis, or some combination of these factors - a premise substantiated by the reduced concentrations of isobutyrate and isovalerate reported by Patra and $\mathrm{Yu}$ (2012). In the present study, the $\mathrm{pH}$ increased linearly with increasing OEO inclusion rate, likely due to the reduction in VFA production, which is in agreement with previous studies (Lin et al., 2012; Patra and $\mathrm{Yu}, 2015 \mathrm{a})$. Several studies have observed that EO supplementation can cause a reduction in VFA concentrations (Busquet et al., 2006; Calsamiglia et al., 2007; Benchaar et al., 2008). Patra and Yu (2012) reported, using an in vitro test, that total VFA concentrations were decreased by adding oregano and clove EO $(0.25,0.50$, and $1.0 \mathrm{~g} / \mathrm{L})$. Additionally, a mixed EO blend (50 or $200 \mathrm{mg} / \mathrm{L}$ eugenol, carvacrol, citral, and cinnamaldehyde oils) fed to $\mathrm{Hu}$ sheep was effective in reducing the acetate:propionate ratio, $\mathrm{NH}_{3}-\mathrm{N}$, and total VFA concentrations (Lin et al., 2013a). As confirmed by the present study, total VFA, acetate, and butyrate concentrations were markedly reduced with increasing OEO inclusion rates, whereas the propionate concentration and acetate:propionate ratio were increased with addition of $52 \mathrm{mg} / \mathrm{L}$ of OEO. Even though VFA concentrations were reduced with increasing OEO inclusion rate, the propionate concentration increased and then decreased in a quadratic response. Inclusion of OEO caused a shift in ruminal fermentation for lower acetate and greater propionate concentrations. Thus, it is plausible that OEO supplementation causes an ionophore-like shift in the ruminal microbial ecology. Several studies have demonstrated that some EO have positive effects on VFA concentrations by decreasing acetate production and increasing propionate production (Mohammed et al., 2004; Busquet et al., 2005a; Poudel et al., 2019). Poudel et al. (2019) demonstrated that feeding the same OEO product used in this study resulted in an increase in ruminal propionate concentrations from Holstein calves, along with an increase in Prevotellaceae. In the present study, supplementation with increasing OEO inclusion rates decreased isobutyrate and isovalerate concentrations. These results are in agreement with the findings reported by Pinski et al. (2016), who suggest that different doses of OEO have different potencies to inhibit proteolysis and aminogenesis. Additional studies using HAP bacterial populations need to be conducted to elucidate the effects of OEO on aminogenesis.

\section{Effects on Total Gas and Methane Production}

In the present study, total gas production was linearly decreased by increasing OEO inclusion rate. These results are in agreement with the in vitro results reported by Patra and $\mathrm{Yu}$ (2012) and Cobellis et al. (2016b), that supplementation with cinnamon bark oil, Ceylon cinnamon bark oil, and oregano oil demonstrated a pronounced inhibition of total gas production. The novel finding in our study is an increasing reduction in methane as a percentage of total gas production with increasing OEO inclusion rate. However, Agarwal et al. (2009) found an increase in gas production with addition of 0.33 and $1.0 \mu \mathrm{L} / \mathrm{mL}$ of peppermint oil. These results demonstrate that the OEO type and dose may elicit different results. Numerous studies have reported strong inhibition in methane production by EO (Wang et al., 2009; Knapp et al., 2014; Cobellis et al., 2016b). For example, Macheboeuf et al. (2008) observed a $98 \%$ reduction in methane production using $5 \mathrm{~m} M$ of $\mathrm{OEO}$ or cinnamon EO. Evaluating 5 different EO (clove, eucalyptus, garlic, oregano, and peppermint), Patra and $\mathrm{Yu}$ (2012) reported that methane production linearly decreased with increasing EO doses, with the greatest methane reduction (87\%) using OEO at a dose of 1.0 $\mathrm{g} / \mathrm{L}$. Garlic oil can inhibit in vitro methane production (38.5\%) with a dose of $167 \mu \mathrm{L} / \mathrm{L}$ (Pawer et al., 2014). The finding of Pawer et al. (2014) is an increasing methane reduction as a $\%$ of total gas production with increasing OEO inclusion rate. In the present study, methane production decreased linearly with increasing OEO supplementation, suggesting that OEO inhibits 
methane synthesis. The decrease in methane production with the addition of OEO is similar to the findings of Tekippe et al. (2011). Feeding lactating cows (8 cows) Origanum vulgare L. leaves (500 g/cow per d) led to an approximately $31 \%$ decrease in ruminal methane production. The inhibition of methane production by OEO may be due to indirect or direct inhibition (or both) of methanogens via a decline in $\mathrm{H}_{2}$ production due to reduced acetate and butyrate and more propionate production (Cieslak et al., 2013; Kumar et al., 2014). Shifting ruminal fermentation to more propionate would inhibit hydrogen-producing bacteria, such as Ruminococcus albus, Ruminococcus flavefaciens, and protozoa (Cobellis et al., 2016b). Further studies are needed to elucidate the exact mechanism by which OEO reduces methane production.

\section{Rumen Microbial Community}

In previous studies, very few bacteria genera were analyzed (Fiorentini et al., 2013; Martínez-Fernández et al., 2014; Zhou et al., 2017), which prevented a deeper understanding of the influence of feed additives on ruminal bacterial communities. In the present study, 16S rRNA sequencing was used to comparatively examine OEO influence on the ruminal bacterial community. Ruminant animals have a very diverse bacterial community, especially at the species level, containing about 5,200 OTU (Kim et al., 2011). A similar result (4,410 OTU) was observed in our study. The phyla Firmicutes, Bacteroidetes, and Proteobacteria were the prevalent bacteria in the current study, which agreed with the results of previous studies (Zhou et al., 2017; Stewart et al., 2018; Yan et al., 2018). Antibacterial potency of OEO (containing phenol) may contribute to the antimicrobial activities of OEO (Ultee et al., 2002). Generally, gram-positive bacteria are thought to be more susceptible to EO than are gram-negative bacteria, due to the lack of a protective outer membrane surrounding the cell wall (Patra and $\mathrm{Yu}, 2012$ ). In the present study, although Firmicutes was not affected by OEO. Members of Firmicutes, Selenomonas, and Mitsuokella, gram-positive genera, were linearly decreased by OEO inclusion rates (Table 4). Dialister and Lactobacillus were linearly increased by OEO inclusion rates, suggesting that these bacteria may play a key role in ruminal biohydrogenation (Huws et al., 2011; Patra and Yu., 2015b). In the present study, supplementation with $13 \mathrm{mg} / \mathrm{L}$ of OEO increased the relative abundance of Lachnospiraceae unclassified, and the relative abundances of Lactobacillus were increased by the 3 highest OEO inclusion rates, suggesting that these bacteria may play a key role in ruminal biohydrogenation (Huws et al., 2011; Patra and Yu., 2015b).
Therefore, changes of microbial compositions via OEO inclusion may be associated with changes in the ruminal biohydrogenation process (Ramos-Morales et al., 2013). Thoetkiattikul et al. (2013) reported that Bacteroidetes was a major non-cellulosic plant constituent degrader in the rumen. Most Bacteroidetes bacterial strains are hemicellulolytic, proteolytic, or amylolytic (Evans et al., 2011). The relative abundance of the top 2 most abundant bacteria of the Bacteroidetes family (Prevotella and Dialister) were increased by OEO, possibly due to reduced competition from other bacteria that are inhibited by OEO (Patra and Yu, 2015b). Prevotella, a gram-negative genus of Bacteroidetes, linearly increased with OEO inclusion rates, maybe due to reduced competition from other bacteria, which are inhibited by OEO (Patra and Yu, 2015b). In addition, we observed that Prevotella was the dominant genus among all treatments, in accordance with some previous in vivo studies (Jami et al., 2013; Thoetkiattikul et al., 2013; Paz et al., 2016). It has been suggested that EO could reduce ruminal protein degradation and ammonia concentrations (McIntosh et al., 2003; Patra, 2011). We also report that OEO supplementation significantly lowered ammonia nitrogen, which is in agreement with previous studies that reported that EO decreased ruminal ammonia concentrations (Patra and $\mathrm{Yu}, 2012$; Patra and Yu, 2014). The methanogens Euryarchaeota are a phylum belonging to the archaea community; in the current study, Euryarchaeota showed very low relative abundance and were decreased at all levels of OEO inclusion, consistent with the decrease of $\mathrm{CH}_{4}$ production in OEO treatments. These results indicate that ruminal methane production may be much more influenced by the relative abundance of archaea, rather than by the microbial population structure (Wallace, 2004; Duarte et al., 2017). We noted that Fibrobacteres accounted for less than $1.0 \%$ of the sequences among all treatments. This result is similar to a study by Patra and $\mathrm{Yu}$ (2015b), reporting 1 OTU Fibrobacteres with the addition of $0.5 \mathrm{~g} / \mathrm{L}$ OEO. In addition, the cellulolytic Fibrobacteres bacteria decreased for the 52 and $91 \mathrm{mg} / \mathrm{L}$ OEO treatments, which may be positively correlated with acetate concentration (Varzaneh et al., 2018). In the present study, OEO supplementation also reduced a few other bacteria, such as Verrucomicrobia, Synergistetes, Spirochaetes, and Planctomycetes. However, rumen metabolism of these genera are not well understood, and further studies are needed to elicit their functions.

\section{CONCLUSIONS}

Previous studies using 16S rRNA analysis demonstrate that OEO supplementation can influence rumi- 
nal bacterial communities to increase digestibility of dry matter and fiber, while shifting fermentation to reduce molar concentrations of acetate and butyrate to increasing propionate. The modulation of fermentation by OEO inclusion rate altered many ruminal bacterial genera that are associated with feed digestibility and ruminal fermentation characteristics. However, a large number of ruminal bacteria remain to be cultured, cautioning that the results obtained in the present study might have limitations. Future in vivo studies are needed to optimize OEO inclusion rate so that effective methane mitigation can be achieved without altering ruminal feed digestion and fermentation. However, an optimal OEO feeding rate for future animal studies appears to be $52 \mathrm{mg} / \mathrm{L}$ for mature ruminants.

\section{ACKNOWLEDGMENTS}

The authors express their appreciation to the farm staff and personnel at the Lintao Huajia Animal Husbandry Co. Ltd. for the care and feeding of the animals. We thank the scientists of the Animal Husbandry, Pasture, and Green Agriculture Institute of Gansu Academy of Agricultural Sciences for their technical assistance. This study was funded by four research grants from National Natural Science Foundation of China (\#31460592, Beijing, China), China's Agricultural Research system (CARS-39-18, Beijing, China), Agroscientific Research in the Public Interest (201503134, Beijing, China), and Gansu Academy of Agricultural Science Fund (2017GAAS30; Lanzhou, China). The authors have not stated any conflicts of interest.

\section{REFERENCES}

Agarwal, N., C. Shekhar, R. Kumar, L. C. Chaudhary, and D. N. Kamra. 2009. Effect of peppermint (Mentha piperita) oil on in vitro methanogenesis and fermentation of feed with buffalo rumen liquor. Anim. Feed Sci. Technol. 148:321-327. https://doi.org/10 $.1016 /$ j.anifeedsci.2008.04.004.

AOAC International. 2016. Official Methods of Analysis. AOAC International, Gaithersburg, MD.

Bakkali, F., S. Averbeck, D. Averbeck, and M. Idaomar. 2008. Biological effects of essential oils-A review. Food Chem. Toxicol. 46:446475. https://doi.org/10.1016/j.fct.2007.09.106.

Baser, K. H. C. 2002. The Turkish Origanum species. Pages 109-126 in Oregano: The Genera Origanum and Lippia. 1st ed. Taylor and Francis Inc, New York, NY.

Benchaar, C., T. A. McAllister, and P. Y. Chouinard. 2008. Digestion, ruminal fermentation, ciliate protozoal populations, and milk production from dairy cows fed cinnamaldehyde, quebracho condensed tannin, or Yucca schidigera saponin extracts. J. Dairy Sci. 91:4765-4777. https://doi.org/10.3168/jds.2008-1338.

Benchaar, C., H. V. Petit, R. Berthiaume, T. D. Whyte, and P. Y. Chouinard. 2006. Effects of addition of essential oils and monensin premix on digestion, ruminal fermentation, milk production, and milk composition in dairy cows. J. Dairy Sci. 89:4352-4364. https: //doi.org/10.3168/jds.S0022-0302(06)72482-1.

Bentayeb, K., P. Vera, C. Rubio, and C. Nerin. 2009. Adaptation of the ORAC assay to the common laboratory equipment and subsequent application to antioxidant plastic films. Anal. Bioanal. Chem. 394:903-910. https://doi.org/10.1007/s00216-009-2766-0.

Burt, S. 2004. Essential oils: Their antibacterial properties and potential applications in foods-A review. Int. J. Food Microbiol. 94:223-253. https://doi.org/10.1016/j.ijfoodmicro.2004.03.022.

Busquet, M., S. Calsamiglia, A. Ferret, P. W. Cardozo, and C. Kamel. 2005a. Effects of cinnamaldehyde and garlic oil on rumen microbial fermentation in a dual flow continuous culture. J. Dairy Sci. 88:2508-2516. https://doi.org/10.3168/jds.S0022-0302(05)72928 $-3$.

Busquet, M., S. Calsamiglia, A. Ferret, and C. Kamel. 2005b. Screening for effects of plant extracts and active compounds of plants on dairy cattle rumen microbial fermentation in a continuous culture system. Anim. Feed Sci. Technol. 123:597-613. https://doi.org/10 .1016/j.anifeedsci.2005.03.008.

Busquet, M., S. Calsamiglia, A. Ferret, and C. Kamel. 2006. Plant extracts affect in vitro rumen microbial fermentation. J. Dairy Sci. 89:761-771. https://doi.org/10.3168/jds.S0022-0302(06)72137-3.

Calsamiglia, S., M. Busquet, P. W. Cardozo, L. Castillejos, and A. Ferret. 2007. Invited review: Essential oils as modifiers of rumen microbial fermentation. J. Dairy Sci. 90:2580-2595. https://doi .org/10.3168/jds.2006-644.

Cardozo, P. W., S. Calsamiglia, A. Ferret, and C. Kamel. 2005. Screening for the effects of natural plant extracts at different $\mathrm{pH}$ on in vitro rumen microbial fermentation of a high-concentrate diet for beef cattle. J. Anim. Sci. 83:2572-2579. https://doi.org/10.2527/ 2005.83112572x.

Chaney, A. L., and E. P. Marbach. 1962. Modified reagents for determination of urea and ammonia. Clin. Chem. 8:130-132.

Cieslak, A., M. Szumacher-Strabel, A. Stochmal, and W. Oleszek. 2013. Plant components with specific activities against rumen methanogens. Animal 7:253-265. https://doi.org/10.1017/ S1751731113000852.

Cobellis, G., M. Trabalza-Marinucci, M. C. Marcotullio, and Z. Yu. 2016b. Evaluation of different essential oils in modulating methane and ammonia production, rumen fermentation, and rumen bacteria in vitro. Anim. Feed Sci. Technol. 215:25-36. https://doi.org/ 10.1016/j.anifeedsci.2016.02.008.

Dragland, S., H. Senoo, K. Wake, K. Holte, and R. Blomhoff. 2003. Several culinary and medicinal herbs are important sources of dietary antioxidants. J. Nutr. 133:1286-1290. https://doi.org/10 $.1093 /$ jn/133.5.1286.

Duarte, A. C., D. B. Holman, T. W. Alexander, Z. Durmic, P. E. Vercoe, and A. V. Chaves. 2017. The type of forage substrate preparation included as substrate in a RUSITEC system affects the ruminal microbiota and fermentation characteristics. Front. Microbiol. 8:704. https://doi.org/10.3389/fmicb.2017.00704.

Durmic, Z., C. S. McSweeney, G. W. Kemp, P. Hutton, R. J. Wallace, and P. E. Vercoe. 2008. Australian plants with potential to inhibit bacteria and processes involved in ruminal biohydrogenation of fatty acids. Anim. Feed Sci. Technol. 145:271-284. https://doi .org/10.1016/j.anifeedsci.2007.05.052.

Edgar, R. C. 2010. Search and clustering order of magnitude faster than BLAST. Bioinformatics 26:2460-2461. https://doi.org/10 .1093/bioinformatics/btq461.

Edgar, R. C., B. J. Haas, J. C. Clemente, C. Quince, and R. Knight. 2011. UCHIME improves sensitivity and speed of chimera detection. Bioinformatics 27:2194-2200. https://doi.org/10.1093/ bioinformatics/btr381.

Evans, N. J., J. M. Brown, R. D. Murray, B. Getty, R. J. Birtles, C. A. Hart, and S. D. Carter. 2011. Characterization of novel bovine gastrointestinal tract Treponema isolates and comparison with bovine digital dermatitis treponemes. Appl. Environ. Microbiol. 77:138-147. https://doi.org/10.1128/AEM.00993-10.

Fadrosh, D. W., B. Ma, P. Gajer, N. Sengamalay, S. Ott, R. M. Brotman, and J. Ravel. 2014. An improved dual-indexing approach for multiplexed 16S rRNA gene sequencing on the Illumina MiSeq platform. Microbiome 2:6. https://doi.org/10.1186/2049-2618-2-6.

Fiorentini, G., J. D. Messana, P. H. M. Dian, R. A. Reis, R. C. Canesin, A. V. Pires, and T. T. Berchielli. 2013. Digestibility, fermentation and rumen microbiota of crossbred heifers fed diets with 
different soybean oil availabilities in the rumen. Anim. Feed Sci. Technol. 181:26-34. https://doi.org/10.1016/j.anifeedsci.2013.01 .011 .

Froehlich, K. A., K. W. Abdelsalam, C. Chase, J. Koppien-Fox, and D. P. Casper. 2017. Evaluation of essential oils and prebiotics for newborn dairy calves. J. Anim. Sci. 95:3772-3782. https://doi.org/ 10.2527/jas.2017.1601

Gerber, P., C. Opio, T. Vellinga, A. Falcucci, G. Tempio, G. Gianni, and T. Robinson. 2013. Greenhouse Gas Emissions from Ruminant Supply Chains: A Global Life Cycle Assessment. Food and Agriculture Organization, Rome, Italy.

Greathead, H. 2003. Plants and plant extracts for improving animal productivity. Proc. Nutr. Soc. 62:279-290. https://doi.org/10 1079/PNS2002197.

Hart, K. J., D. R. Yáñez-Ruiz, S. M. Duval, N. R. McEwan, and C. J. Newbold. 2008. Plant extracts to manipulate rumen fermentation. Anim. Feed Sci. Technol. 147:8-35. https://doi.org/10.1016/ j.anifeedsci.2007.09.007.

He, Z. M., G. P. Li, and D. S. Zhu. 2016. Laboratory animal management and use guidelines. Science Press, Beijing, China.

Hristov, A. N., C. Lee, T. Cassidy, K. Heyler, J. A. Tekippe, G. A. Varga, B. Corl, and R. C. Brandt. 2013. Effect of Origanum vulgare L. leaves on rumen fermentation, production, and milk fatty acid composition in lactating dairy cows. J. Dairy Sci. 96:1189-1202. https://doi.org/10.3168/jds.2012-5975.

Hu, W. L., J. X. Liu, J. A. Ye, Y. M. Wu, and Y. Q. Guo. 2005. Effect of tea saponin on rumen fermentation in vitro. Anim. Feed Sci. Technol. 120:333-339. https://doi.org/10.1016/j.anifeedsci.2005 .02 .029 .

Huws, S. A., E. J. Kim, M. R. Lee, M. B. Scott, J. K. Tweed, E. Pinloche, R. J. Wallace, and N. D. Scollan. 2011. As yet uncultured bacteria phylogenetically classified as Prevotella, Lachnospiraceae incertae sedis and unclassified Bacteroidales, Clostridiales and Ruminococcaceae may play a predominant role in ruminal biohydrogenation. Environ. Microbiol. 13:1500-1512. https://doi.org/10 $.1111 /$ j.1462-2920.2011.02452.x.

Jami, E., A. Israel, A. Kotser, and I. Mizrahi. 2013. Exploring the bovine rumen bacterial community from birth to adulthood. ISME J. 7:1069-1079. https://doi.org/10.1038/ismej.2013.2.

Joch, M., L. Cermak, J. Hakl, B. Hucko, D. Duskova, and M. Marounek. 2016. In vitro screening of essential oil active compounds for manipulation of rumen fermentation and methane mitigation. Asian-Australas. J. Anim. Sci. 29:952. https://doi.org/10.5713/ ajas.15.0474.

Kamra, D. N., N. Agarwal, and L. C. Chaudhary. 2006. Inhibition of ruminal methanogenesis by tropical plants containing secondary compounds. International Congress Series: Excerpta Medica. 1293:156-163. https://doi.org/10.1016/j.ics.2006.02.002.

Kim, M., M. Morrison, and Z. Yu. 2011. Status of the phylogenetic diversity census of ruminal microbiomes. FEMS Microbiol. Ecol. 76:49-63. https://doi.org/10.1111/j.1574-6941.2010.01029.x.

Knapp, J. R., G. L. Laur, P. A. Vadas, W. P. Weiss, and J. M. Tricarico. 2014. Invited review: Enteric methane in dairy cattle production: Quantifying the opportunities and impact of reducing emissions. J. Dairy Sci. 97:3231-3261. https://doi.org/10.3168/jds .2013-7234.

Kumar, S., P. K. Choudhury, M. D. Carro, G. W. Griffith, S. S. Dagar, M. Puniya, S. Calabro, S. R. Ravella, T. Dhewa, R. C. Upadhyay, S. K. Sirohi, S. S. Kundu, M. Wanapat, and A. K. Puniya. 2014. New aspects and strategies for methane mitigation from ruminants. Appl. Microbiol. Biotechnol. 98:31-44. https://doi.org/10 .1007/s00253-013-5365-0.

Lin, B., Y. Lu, A. Z. M. Salem, J. H. Wang, Q. Liang, and J. X. Liu. 2013b. Effects of essential oil combinations on sheep ruminal fermentation and digestibility of a diet with fumarate included. Anim. Feed Sci. Technol. 184:24-32. https://doi.org/10.1016/j .anifeedsci.2013.05.011.

Lin, B., Y. Lu, J. H. Wang, Q. Liang, and J. X. Liu. 2012. The effects of combined essential oils along with fumarate on rumen fermentation and methane production in vitro. Anim. Feed Sci. Technol. 21:198-210. https://doi.org/10.22358/jafs/66064/2012.
Lin, B., Z. Tan, G. Xiao, M. Wang, Z. Cong, S. Wang, S. Tang, C. S. Zhou, Z. H. Sun, and W. Wang. 2009. Evaluation of compositional and nutritional equivalence of genetically modified rice to conventional rice using in situ and in vitro techniques. J. Sci. Food Agric. 89:1490-1497. https://doi.org/10.1002/jsfa.3613.

Lin, B., J. H. Wang, Y. Lu, Q. Liang, and J. X. Liu. 2013a. In vitro rumen fermentation and methane production are influenced by active components of essential oils combined with fumarate. J. Anim. Physiol. Anim. Nutr. (Berl.) 97:1-9. https://doi.org/10 $.1111 /$ j.1439-0396.2011.01236.x.

Liu, T. H. Chen, D. P. Casper, and J. P. Wu. 2017. Impact of essential oils on the growth performance of newborn Holstein calves. J. Anim. Sci. 95(suppl_4):371-372. https://doi.org/10.2527/asasann .2017 .868 .

Lourenço, M., P. W. Cardozo, S. Calsamiglia, and V. Fievez. 2008. Effects of saponins, quercetin, eugenol, and cinnamaldehyde on fatty acid biohydrogenation of forage polyunsaturated fatty acids in dual-flow continuous culture fermenters. J. Anim. Sci. 86:3045-3053. https://doi.org/10.2527/jas.2007-0708.

Macheboeuf, D., D. P. Morgavi, Y. Papon, J. L. Mousset, and M. Arturo-Schaan. 2008. Dose-response effects of essential oils on in vitro fermentation activity of the rumen microbial population. Anim. Feed Sci. Technol. 145:335-350. https://doi.org/10.1016/j .anifeedsci.2007.05.044.

Magoč, T., and S. L. Salzberg. 2011. FLASH: Fast length adjustment of short reads to improve genome assemblies. Bioinformatics 27:2957-2963. https://doi.org/10.1093/bioinformatics/btr507.

Martínez-Fernández, G., L. Abecia, E. Ramos-Morales, A. I. MartinGarcía, E. Molina-Alcaide, and D. R. Yáñez-Ruiz. 2014. Effects of propyl propane thiosulfinate on nutrient utilization, ruminal fermentation, microbial population and methane emissions in goats. Anim. Feed Sci. Technol. 191:16-25. https://doi.org/10.1016/j .anifeedsci.2014.01.008.

Matsuura, H., H. Chiji, C. Asakawa, M. Amano, T. Yoshihara, and J. Mizutani. 2003. DPPH radical scavengers from dried leaves of oregano (Origanum vulgare L.). Biosci. Biotechnol. Biochem. 67:2311-2316. https://doi.org/10.1271/bbb.67.2311.

McIntosh, F. M., P. Williams, R. Losa, R. J. Wallace, D. A. Beever, and C. J. Newbold. 2003. Effects of essential oils on ruminal microorganisms and their protein metabolism. Appl. Environ. Microbiol. 69:5011-5014. https://doi.org/10.1128/AEM.69.8.5011-5014.2003.

Menke, K. H., and H. Steingass. 1988. Estimation of the energetic feed value obtained from chemical analysis and in vitro gas production using rumen fluid. Anim. Res. Dev. 28:7-55.

Meyer, N. F., G. E. Erickson, T. J. Klopfenstein, M. A. Greenquist, M. K. Luebbe, P. Williams, and M. A. Engstrom. 2009. Effect of essential oils, tylosin, and monensin on finishing steer performance, carcass characteristics, liver abscesses, ruminal fermentation, and digestibility. J. Anim. Sci. 87:2346-2354. https://doi.org/10.2527/ jas.2008-1493.

Mitra, S., M. Stärk, and D. H. Huson. 2011. Analysis of 16S rRNA environmental sequences using MEGAN. BMC Genomics 12(Suppl 3):S17. https://doi.org/10.1186/1471-2164-12-S3-S17 https://doi .org/10.1186\%2F1471-2164-12-S3-S17.

Mohammed, N., N. Ajisaka, Z. A. Lila, K. Hara, K. Mikuni, K. Hara, S. Kanda, and H. Itabashi. 2004. Effect of Japanese horseradish oil on methane production and ruminal fermentation in vitro and in steers. J. Anim. Sci. 82:1839-1846. https://doi.org/10.2527/2004 $.8261839 x$

Molero, R., M. Ibars, S. Calsamiglia, A. Ferret, and R. Losa. 2004. Effects of a specific blend of essential oil compounds on dry matter and crude protein degradability in heifers fed diets with different forage to concentrate ratios. Anim. Feed Sci. Technol. 114:91-104. https://doi.org/10.1016/j.anifeedsci.2003.11.011.

NRC. 2007. Nutrient requirements of small ruminants: Sheep, goats, cervids, and New World camelids. Natl. Acad. Press, Washington, DC. https://doi.org/10.17226/11654.

Patra, A. K. 2011. Effects of essential oils on rumen fermentation, microbial ecology and ruminant production. Asian J. Anim. Vet. Adv. 6:416-428. https://doi.org/10.3923/ajava.2011.416.428. 
Patra, A. K., and Z. Yu. 2012. Effects of essential oils on methane production, fermentation, abundance and diversity of rumen microbial populations. Appl. Environ. Microbiol. 78:4271-4280. https:/ /doi.org/10.1128/AEM.00309-12.

Patra, A. K., and Z. Yu. 2014. Effects of vanillin, quillaja saponin, and essential oils on in vitro fermentation and protein-degrading microorganisms of the rumen. Appl. Microbiol. Biotechnol. 98:897905. https://doi.org/10.1007/s00253-013-4930-x.

Patra, A. K., and Z. Yu. 2015a. Effects of adaptation of in vitro rumen culture to garlic oil, nitrate, and saponin and their combinations on methanogenesis, fermentation, and abundances and diversity of microbial populations. Front. Microbiol. 6:1434. https://doi.org/ 10.3389 /fmicb.2015.01434.

Patra, A. K., and Z. Yu. 2015b. Essential oils affect populations of some rumen bacteria in vitro as revealed by microarray (RumenBactArray) analysis. Front. Microbiol. 6:297. https://doi.org/10 $.3389 /$ fmicb.2015.00297.

Paz, H. A., C. L. Anderson, M. J. Muller, P. J. Kononoff, and S. C. Fernando. 2016. Rumen bacterial community composition in Holstein and Jersey cows is different under same dietary condition and is not affected by sampling method. Front. Microbiol. 7:1206. https://doi.org/10.3389/fmicb.2016.01206.

Pinski, B., M. Günal, and A. A. AbuGhazaleh. 2016. The effects of essential oil and condensed tannin on fermentation and methane production under in vitro conditions. Anim. Prod. Sci. 56:17071713. https://doi.org/10.1071/AN15069.

Poudel, P., K. Froehlich, D. P. Casper, and B. St-Pierre. 2019. Feeding essential oils to neonatal Holstein dairy calves results in increased ruminal Prevotellaceae abundance and propionate concentrations. Microorganisms 7:120. https://doi.org/10.3390/ microorganisms 7050120

Priest, F. G. 1977. Extracellular enzyme synthesis in the genus Bacillus. Bacteriol. Rev. 41:711-753.

Ramos-Morales, E., G. Martínez-Fernández, L. Abecia, A. I. MartinGarcía, E. Molina-Alcaide, and D. R. Yáñez-Ruiz. 2013. Garlic derived compounds modify ruminal fatty acid biohydrogenation and induce shifts in the Butyrivibrio community in continuous-culture fermenters. Anim. Feed Sci. Technol. 184:38-48. https://doi.org/ 10.1016/j.anifeedsci.2013.05.017.

R Core Team. 2017. R: A language and environment for statistical computing. R Foundation for Statistical Computing, Vienna, Austria.

Sallam, S. M. A., I. C. S. Bueno, P. Brigide, P. B. Godoy, D. M. S. S. Vitti, and A. L. Abdalla. 2009. Efficacy of eucalyptus oil on in vitro ruminal fermentation and methane production. Options Méditerranéennes. 85:267-272. http://om.ciheam.org/article.php ?IDPDF $=801016$

Simitzis, P. E. 2017. Enrichment of animal diets with essential oils-A great perspective on improving animal performance and quality characteristics of the derived products. Medicines (Basel) 4:35. https://doi.org/10.3390/medicines4020035.

Sivropoulou, A., E. Papanikolaou, C. Nikolaou, S. Kokkini, T. Lanaras, and M. Arsenakis. 1996. Antimicrobial and cytotoxic activities of Origanum essential oils. J. Agric. Food Chem. 44:1202-1205. https: //doi.org/10.1021/jf950540t.

Stewart, R. D., M. D. Auffret, A. Warr, A. H. Wiser, M. O. Press, K. W. Langford, I. Liachko, T. J. Snelling, R. J. Dewhurst, A. W. Walker, R. Roehe, and M. Watson. 2018. Assembly of 913 microbial genomes from metagenomic sequencing of the cow rumen. Nat. Commun. 9:870. https://doi.org/10.1038/s41467-018-03317-6.

Tager, L. R., and K. M. Krause. 2011. Effects of essential oils on rumen fermentation, milk production, and feeding behavior in lactating dairy cows. J. Dairy Sci. 94:2455-2464. https://doi.org/10 $.3168 / \mathrm{jds} .2010-3505$.

Tekippe, J. A., A. N. Hristov, K. S. Heyler, T. W. Cassidy, V. D. Zheljazkov, J. F. S. Ferreira, S. K. Karnati, and G. A. Varga. 2011. Rumen fermentation and production effects of Origanum vulgare
L. leaves in lactating dairy cows. J. Dairy Sci. 94:5065-5079. https: //doi.org/10.3168/jds.2010-4095.

Tekippe, J. A., A. N. Hristov, K. S. Heyler, V. D. Zheljazkov, J. Ferreira, and G. A. Varga. 2010. Effects of herbs and essential oils on in vitro batch culture ruminal fermentation. J. Dairy Sci. 93:175175 .

Thoetkiattikul, H., W. Mhuantong, T. Laothanachareon, S. Tangphatsornruang, V. Pattarajinda, L. Eurwilaichitr, and V. Champreda 2013. Comparative analysis of microbial profiles in cow rumen fed with different dietary fiber by tagged $16 \mathrm{~S}$ rRNA gene pyrosequencing. Curr. Microbiol. 67:130-137. https://doi.org/10.1007/s00284 $-013-0336-3$

Ultee, A., M. H. J. Bennik, and R. Moezelaar. 2002. The phenolic hydroxyl group of carvacrol is essential for action against the food-borne pathogen Bacillus cereus. Appl. Environ. Microbiol. 68:1561-1568. https://doi.org/10.1128/AEM.68.4.1561-1568.2002.

Van Soest, P. J., J. B. Robertson, and B. A. Lewis. 1991. Methods for dietary fiber, neutral detergent fiber, and nonstarch polysaccharides in relation to animal nutrition. J. Dairy Sci. 74:3583-3597. https://doi.org/10.3168/jds.S0022-0302(91)78551-2.

Varzaneh, M. B., F. Klevenhusen, Q. Zebeli, and R. Petri. 2018. Scrophularia striata extract supports rumen fermentation and improves microbial diversity in vitro compared to monensin. Front. Microbiol. 9:1-9. https://doi.org/10.3389/fmicb.2018.02164.

Wallace, R. J. 2004. Antimicrobial properties of plant secondary metabolites. Proc. Nutr. Soc. 63:621-629. https://doi.org/10.1079/ PNS2004393.

Wallace, R. J., N. R. McEwan, F. M. McIntosh, B. Teferedegne, and C. J. Newbold. 2002. Natural products as manipulators of rumen fermentation. Asian-Australas. J. Anim. Sci. 15:1458-1468. https: //doi.org/10.5713/ajas.2002.1458.

Wang, C. J., S. P. Wang, and H. Zhou. 2009. Influences of flavomycin, ropadiar, and saponin on nutrient digestibility, rumen fermentation, and methane emission from sheep. Anim. Feed Sci. Technol. 148:157-166. https://doi.org/10.1016/j.anifeedsci.2008.03.008.

Wemheuer, B., S. Güllert, S. Billerbeck, H. A. Giebel, S. Voget, M. Simon, and R. Daniel. 2014. Impact of a phytoplankton bloom on the diversity of the active bacterial community in the southern North Sea as revealed by metatranscriptomic approaches. FEMS Microbiol. Ecol. 87:378-389. https://doi.org/10.1111/1574-6941 .12230

Yan, X. T., B. Y. Yan, Q. M. Ren, J. J. Dou, W. W. Wang, J. J. Zhang, J. W. Zhou, R. J. Long, L. M. Ding, J. Han, Z. P. Li, and Q. Qiu. 2018. Effect of slow-release urea on the composition of ruminal bacteria and fungi communities in yak. Anim. Feed Sci. Technol. 244:18-27. https://doi.org/10.1016/j.anifeedsci.2018.07 .016 .

Yáñez-Ruiz, D. R., A. Bannink, J. Dijkstra, E. Kebreab, D. P. Morgavi, P. O'Kiely, C. K. Reynolds, A. Schwarm, K. J. Shingfield, Z Yu, and A. N. Hristov. 2016. Design, implementation and interpretation of in vitro batch culture experiments to assess enteric methane mitigation in ruminants-A review. Anim. Feed Sci. Technol. 216:1-18. https://doi.org/10.1016/j.anifeedsci.2016.03.016.

Yang, W. Z., C. Benchaar, B. N. Ametaj, A. V. Chaves, M. L. He, and T. A. McAllister. 2007. Effects of garlic and juniper berry essential oils on ruminal fermentation and on the site and extent of digestion in lactating cows. J. Dairy Sci. 90:5671-5681. https://doi.org/ 10.3168/jds.2007-0369.

Zeng, B., S. Han, P. Wang, B. Wen, W. Jian, W. Guo, Z. Yu, D. Du, X. Fu, F. Kong, M. Yang, X. Si, J. Zhao, and Y. Li. 2015. The bacterial communities associated with fecal types and body weight of rex rabbits. Sci. Rep. 5:9342. https://doi.org/10.1038/srep09342.

Zhou, Z., L. Fang, Q. Meng, S. Li, S. Chai, S. Liu, and J. T. Schonewille. 2017. Assessment of ruminal bacterial and archaeal community structure in yak (Bos grunniens). Front. Microbiol. 8:179. https://doi.org/10.3389/fmicb.2017.00179. 